\title{
Tratamiento de efluentes acuosos provenientes de la industria de biodiesel, utilizando tecnología de membranas
}

\author{
Treatment of aqueous effluents from \\ the biodiesel industry using \\ membrane technology
}

Leticia Raquel Firman ${ }^{1}$, Nelio Ariel Ochoa ${ }^{2}$, José Marchese $^{2}$, Cecilia Liliana Pagliero ${ }^{1}$

\footnotetext{
${ }^{1}$ Departamento de Tecnología Química-Facultad de Ingeniería (UNRC-CONICET), Ruta 36 Km 601. 5800 Río Cuarto, Buenos Aires, Buenos Aires, Argentina. Argentina. e-mail: lfirman@ing.unrc.edu.ar, cpagliero@ing.unrc.edu.ar ${ }^{2}$ Instituto de Física Aplicada - INFAP (UNSL-CONICET), San Luis, San Luis, Argentina. e-mail: aochoa@unsl.edu.ar, marchese@unsl.edu.ar
}

\section{RESUMEN}

Los efluentes acuosos provenientes de la producción de biodiesel son generados principalmente a partir del proceso de lavado, etapa en la cual se eliminan contaminantes para asegurar que el biodiesel cumpla con las normas internacionales. Estos efluentes contienen impurezas de la mezcla de esteres metílicos, aceite residual, mono y diglicéridos, sales solubles, catalizador, jabones, glicerol y alcohol, por lo que contienen una elevada carga contaminante.

El objetivo del presente trabajo es el desarrollo de la tecnología de membranas para el tratamiento de efluentes acuosos provenientes de la industria de biodiesel. Se sintetizó una membrana de ultrafiltración (UF) (17\% de polifluoruro de vinilideno -PVDF-, 8,5\% polivinilpirrolidona -PVP- k30) por el método de inversión de fase. Las experiencias se realizaron con una celda de flujo cruzado. Las condiciones operacionales fueron: $\mathrm{T}=$ $20^{\circ} \mathrm{C}, \Delta \mathrm{p}=0,4-1$ bar y v=0,5-1 m/s. Se utilizó como alimentación tanto una muestra sintética preparada en el laboratorio como una muestra real de efluente proveniente de una industria de biodiesel.

Se observó que a un caudal de alimentación constante, el flujo permeado disminuye al aumentar la presión. Dicho comportamiento es atribuido al ensuciamiento de la membrana (región controlada por la transferencia de masa). Mientras que al aumentar la velocidad de permeación a presión constante, el flujo permeado aumenta gradualmente, ya que al aumentar la velocidad se logra limpiar la superficie de la membrana obteniendo un menor ensuciamiento.

A partir de la caracterización de la alimentación y el permeado, se encontró que la membrana estudiada retiene entre el $89-100 \%$ de aceites y grasas, y entre $20-40 \%$ de la demanda química de oxígeno (DQO) presentes en el efluente, $\mathrm{pH}$ y sólidos solubles totales (TSS) permanecieron invariables.

Palabras clave: Tratamiento de Efluente, Biodiesel, Aguas Residuales, Ultrafiltración.

\section{ABSTRACT}

The wastewaters from biodiesel production are generated mainly in the washing process, in this step the contaminants of biodiesel are eliminated in order to the obtained biodiesel meets with the internationals regulations. These wastes contain impurities such as methyl ester, residual oil, mono and diglycerides, soluble salts, catalyst, soap, glycerol and alcohol.

The aim of this study is the application of membrane technology for the treatment of aqueous wastes from biodiesel industry. By means of the phase inversion method was synthesized a membrane of UF using a $17 \mathrm{wt} \%$ of polyvinylidene polyfluoride (PVDF) and $8,5 \mathrm{wt} \%$ polyvinylpyrrolidone (PVP) casting solution. The filtration essays were performed in a cross flow cell. The operational conditions were: $\mathrm{T}=20^{\circ} \mathrm{C}, \Delta \mathrm{p}=0.4-$ 
1 bar y v $=0.5-1 \mathrm{~m} / \mathrm{s}$. A synthetic sample prepared in the laboratory and a real effluent sample from biodiesel industry was employed as membrane feed.

At constant feed stream speed, the permeate flux decreases with increase of pressure (mass transfer controlled region). While an increasing in the permeation speed at constant pressure the permeate flux gradually increases. With increasing speed, membrane surface cleaning increases due to solutes are swept tangentially away.

It was concluded that the studied membrane retains between $83-100 \%$ of oils and fats, and between $20-40 \%$ of DQO, whereas $\mathrm{pH}$ and TSS remain constant.

Keywords: Effluent Treatment, Biodiesel, Wastewater, Ultrafiltration

\section{INTRODUCCIÓN}

El biodiesel (BD) es un combustible compuesto por monoalquil ésteres de ácidos grasos de cadena larga derivados de materias primas renovables, tales como aceites vegetales o grasas animales.

En Argentina se utiliza el aceite crudo de soja para la obtención de BD, debido a que es la principal fuente de aceite que se puede encontrar en éste país en gran cantidad.

El crecimiento del BD en los últimos años ha sido notable. En Argentina y la Unión Europea la capacidad productiva de biodiesel de soja pasó de 6,2 millones de toneladas en el 2006 a 23,1 millones de toneladas en el 2010 [1].

El biodiesel se produce generalmente por una reacción de transesterificación. En este proceso los triglicéridos reaccionan con un alcohol de cadena corta (metanol o etanol son los más utilizados debido a su bajo costo y a sus adecuadas propiedades), en presencia de un catalizador, para dar como resultado biodiesel (metil o etil ésteres) y glicerol [2]. Luego de la reacción, el biodiesel crudo presenta un alto contenido de impurezas tales como jabones, catalizador, agua, metales, alcohol y glicerol libre, que deben ser eliminados en posteriores etapas de purificación. El proceso de purificación convencional consiste en lavar el biodiesel crudo para eliminar las impurezas presentes. Este proceso genera una gran cantidad de aguas residuales altamente contaminante, lo que provoca efectos ambientales, debido a los altos contenidos de demanda química de oxígeno (DQO), demanda biológica de oxígeno (DBO) y valores de $\mathrm{pH}$ elevados [3].Se ha reportado que cada $100 \mathrm{~L}$ de biodiesel producido, se generan más de $20 \mathrm{~L}$ de aguas residuales[4]. En la actualidad existen diferentes métodos para los tratamientos de estas mezclas acuosas (mecánicos, químicos y térmicos). Sin embargo, estos métodos convencionales presentan ciertas desventajas tales como: 1) baja eficiencia de eliminación de contaminantes, 2) dificultades de funcionamiento y 3) alto costo operativo [5].

Algunos autores han estudiado el tratamiento de este tipo de efluentes mediante: tratamiento biológico [4,6], coagulación y/o electrocoagulación [7-10] y adsorción [11,12]. Este último método casi no ha sido utilizado para el tratamiento de aguas residuales de biodiesel. El tratamiento biológico es bastante limitado, debido a que el contenido de sólidos presentes en las aguas residuales de biodiesel es bastante alto, lo que inhibe el crecimiento de microorganismos y reduce los rendimientos de eliminación de tratamiento biológico [8]. SHARMA et al. [13] consideran que el proceso biológico por sí solo no es adecuado para el tratamiento de efluentes acuosos de biodiesel.

Ngamlerdpokin et al. [10] aseguran que el proceso de coagulación es simple y económico, pero presenta como desventaja un efluente de menor calidad frente a un efluente tratado con electrocoagulación. Los autores han demostrado que con la coagulación química se logra reducir los valores de DQO en $\sim 79 \%$ mientras que con electrocoagulación la reducción es de $\sim 99 \%$.

En los últimos años se han realizado muchos esfuerzos para desarrollar una técnica alternativa que reemplace los procesos convencionales antes mencionados. Uno de los procesos que se ha desarrollado recientemente es la tecnología de membrana para ser aplicada en procesamiento de efluentes acuosos derivados de emulsiones oleosas. En comparación con otros procesos de separación, el proceso de tecnología de membranas posee varias características únicas, tales como: elevadas retenciones y condiciones operacionales moderadas (de temperatura y de presión) [14].

Los procesos de separación por membrana utilizados para el tratamiento de aguas residuales, impulsados por presión, son: microfiltración (MF) (0,5-3 bar), ultrafiltración (UF) (1-10 bar), nanofiltración (NF) (530 bar), ósmosis inversa (RO) (10-50 bar). A medida que se reduce el tamaño de poro, la resistencia a la transferencia de materia se hace mayor, por lo que la presión aplicada debe aumentar.

Diferentes estudios han informado que la aplicación de microfiltración y ultrafiltración (MF-UF) son procesos eficientes y adecuados en el procesamiento de emulsiones oleosas provenientes de una industria metalmecánica $[15,16]$. 
El uso de la tecnología de membranas en el tratamiento brinda importantes ventajas. En general, se produce la separación a temperatura ambiente y posee bajos costos operativos. Sin embargo, este tipo de procesos presentan como desventaja la rápida disminución del flujo permeado debido al ensuciamiento de la membrana.

Si bien el corazón de la investigación en la tecnología de membranas es la formulación de las membranas en sí mismas, poco se ha realizado respecto al material y tipo de membranas utilizadas. Tanto el material como el tipo de membrana son los factores responsables de un fenómeno indeseable durante los procesos de membrana, el ensuciamiento, limitando la eficiencia del método. Los materiales más comúnmente utilizados son polifluoruro de vinilideno (PVDF), polietersulfona (PES), polisulfona (PSf), poliacetato de celulosa (PAC) entre otros, y los tipos de membranas pueden ser planas, espiraladas y de fibras huecas. La adsorción de solutos involucra generalmente interacciones hidrofóbicas, siendo por lo tanto las membranas hidrofóbicas aquellas que poseen una mayor susceptibilidad al ensuciamiento. Sin embargo estas membranas, son usadas para el tratamiento de efluentes en razón de sus superiores características de estabilidad térmica y química. De aquí que el salto cuantitativo para la recuperación efectiva de sustancias nocivas para el medio ambiente estará dado por aquellas membranas que permitan mantener sus características de estabilidad y que disminuyan considerablemente el ensuciamiento.

Pocos han sido los autores que han investigado el tratamiento de efluente proveniente de la industria de biodiesel, utilizando tecnologías separativas de membranas. LOW et al. [5] compararon el comportamiento de membranas de MF-UF, permeando a través de ellas una mezcla obtenida de lavar derivados de éster metílico de palma (PME) con agua durante los procesos de purificación del producto de la transesterificación de biodiesel catalizada con hidróxido de sodio. Emplearon una membrana de MF de acetato de celulosa, plana de 0,45 micrones (Advantec MFS) y otra membrana polimérica plana de UF de 0,05 micrones (New Logic International Inc.). Utilizaron un módulo de membrana de flujo cruzado de DISARM Tami Industries. La presión de transmembrana varió de 1,5-4,5 psi, se realizaron experiencias a dos velocidades de flujo diferentes $(1,3 \mathrm{~m} / \mathrm{min}$ y $1,5 \mathrm{~m} / \mathrm{min})$ y la temperatura de la mezcla se mantuvo constante a $30^{\circ} \mathrm{C}$. Ellos concluyen que la membrana de UF tiene, a bajos valores de presión de transmembrana, una mayor permeación de metil éster y mejor retención de jabón, que la membrana de MF.

SHIRAZI et al. [14] llevaron a cabo un proceso de MF. Utilizaron una membrana de PS y una membrana de PS modificada, con el fin de mejorar la morfología de la superficie, reducir el tamaño medio de poro, reducir parámetros de rugosidad y aumentar la hidrofobicidad. Para llevar a cabo las experiencias de permeación utilizaron un módulo de extremo cerrado. Los ensayos se realizaron a temperatura ambiente a 0,4 bar. A partir de la aplicación de la membrana de PS, obtuvieron una reducción de 58, 26, 92 y 95 de DQO, DBO, sólidos totales (TS) y TSS. Mientras que con la membrana modificada obtuvieron resultados más prometedores (75, 55, 92 y 96 de DQO, DBO, TS y TSS).

Evidentemente el uso de membranas puede ayudar a obtener reducciones del consumo de agua en el lavado del biodiesel, recuperando el agua tratada (permeado). Por lo tanto el objetivo de este trabajo es el desarrollo y la aplicación de una membrana de UF en el tratamiento de aguas residuales provenientes de la purificación del biodiesel, reduciendo el contenido de aceites y grasas en el efluente y el costo ambiental del manejo del mismo.

\section{MATERIALES Y MÉTODOS}

\subsection{Síntesis y caracterización de membranas}

La membrana UF fue preparada mediante la técnica de inversión de fase [16]. La solución (100 g) preparada consistió de $17 \%$ p/p de polifluoruro de vinilideno (SOLEF 1015 suministrado por Solvay (Bruselas, Bélgica) y $8,5 \%$ p/p polivinilpirrolidona $\mathrm{k} 30$ en N, N-dimetilformamida (DMF) (adquirida en Aldrich (Buenos Aires Argentina). Ésta solución polimérica se extendió sobre un soporte polimérico no-tejido $(20$ x $30 \mathrm{~cm})$ Viledon 2431 (provisto por Carl Freudenberg (Weinheim, Alemania)) a $25^{\circ} \mathrm{C}$ utilizando un extensor de película. A continuación la membrana fue sumergida en un baño de agua bidestilada y luego fue transferida a otro baño de agua destilada durante $24 \mathrm{~h}$.

La técnica de microscopía por barrido electrónico (SEM) permite la obtención de imágenes de la superficie y/o de cortes transversales y/o longitudinales. En este trabajo se pudo observar la morfología de la superficie de la membrana usando un microscopio por barrido electrónico (SEM) LEO 1450VP. 


\subsection{Materia prima}

Para llevar a cabo las experiencias de permeación, se utilizaron dos muestras como alimentación: una muestra preparada en nuestro laboratorio y una muestra real.

La muestra real de efluente de biodiesel fue suministrada por la empresa AOM (Advanced Organic Material S.A., Pilar, Argentina). En base a la información brindada por dicha empresa, con respecto a la composición del efluente real, se preparo un efluente con el fin de mantener sin variaciones las características de la muestra alimentada a la celda de permeación. La composición de la muestra preparada y la muestra real fueron de similares concentraciones de metanol $(\sim 2 \% \mathrm{p} / \mathrm{p})$, sales (fosfato de sodio) $(\sim 1 \% \mathrm{p} / \mathrm{p})$, glicerina $(\sim 5$ $\% \mathrm{p} / \mathrm{p})$, jabones (oleato de sodio) $(\sim 1 \% \mathrm{p} / \mathrm{p})$ y agua $(\sim 91 \% \mathrm{p} / \mathrm{p})$.

\subsection{Determinaciones Analíticas}

Tanto la alimentación como la calidad del permeado y retenido, fueron analizados por técnicas de Demanda Química de Oxigeno (DQO) (Standar Method 5220D), Sólidos Solubles Totales (SST) y contenido de Grasas y Aceites (G\&A) (Método de partición gravimétrica líquido/líquido, Standar Method 5520B). Además se determinó $\mathrm{pH}$.

\subsection{Experiencias de permeación}

Todas las experiencias se llevaron a cabo en una celda de flujo cruzado con un área efectiva de membrana de $6,9 \times 10^{-3} \mathrm{~m}^{2}$ [18]. La solución de alimentación fue bombeada en la membrana por una bomba peristáltica.

El flujo de agua permeado $\mathrm{J}\left(\mathrm{Lm}^{-2} \mathrm{~h}^{-1}\right)$ se determino a partir de la ecuación (1).

$$
J=\frac{1}{A} \frac{V}{t}
$$

La permeabilidad al agua, se calculó a partir la ley de Darcy, mediante la medición del volumen de agua permeada en función de la presión transmembrana.

$$
L_{h}=\frac{J}{\Delta p}
$$

donde $\mathrm{V}$ es el volumen de permeado acumulado durante el tiempo t y $\mathrm{A}$ es el área efectiva de la membrana.

Las condiciones experimentales utilizadas durante la permeación del efluente fueron: temperatura de $20^{\circ} \mathrm{C}$, la presión de transmembrana se varió entre $0,4-1$ bar y la velocidad lineal de flujo entre $0,5-1 \mathrm{~m} / \mathrm{s}$. Se alimentaron aproximadamente $2 \mathrm{~L}$ de efluente en cada experiencia y cada prueba se llevó a cabo durante 90 min con reciclo total del retenido. Para cada experiencia la presión y el caudal de alimentación (velocidad lineal) se mantuvieron constantes mediante el controlador de la bomba peristáltica (Master Flex 77601-10) y una válvula de estrangulación. Durante las experiencias, se determinó el volumen de permeado recogido y se calculó el flujo permeado acumulado $\mathrm{J}\left(\mathrm{Lm}^{-2} \mathrm{~h}^{-1}\right)$ versus el tiempo medio de la ecuación (1). Todos los ensayos de permeación se llevaron por duplicado.

El porcentaje de retención de G\&A y de DQO se calculó como:

$$
\% R=\left(1-\frac{C_{p}}{C_{a}}\right) * 100
$$

donde Cp y Ca son el contenido de G\&A o de DQO en el permeado y alimentación respectivamente (\% p/p).

Luego de cada experiencia de UF, la membrana sucia se limpió con 2 L de solución de detergente $2 \%$ (p/p) durante 30 minutos, seguido de enjuague con $2 \mathrm{~L}$ agua destilada durante 30 minutos. Finalmente se corroboró la permeabilidad hidráulica de la membrana. En caso que fuese necesario, el procedimiento de limpieza se repitió hasta que la permeabilidad hidráulica de la membrana limpia estuviese dentro del 95-100\% a la de la membrana inicial. 


\section{RESULTADOS Y DISCUSIÓN}

\subsection{Micrografía SEM}

En la Figura 1-a) se muestra la superficie de la membrana asimétrica (MA), en la cual se puede observar cierta rugosidad con poros de similar diámetro distribuidos por toda la superficie, arrojando un valor de radio medio de poro de $\sim 19 \mathrm{~nm}$, el cual fue calculado mediante las imágenes de la superficie de la membrana y un software operativo).

En la Figura 1-b) se muestra una micrografía de la sección transversal de la MA, en la cual se pueden observar dos regiones bien definidas: una capa de piel fina (selectiva) sobre la parte superior y debajo de ella una subestructura altamente porosa de macrovacíos tipo dedos.
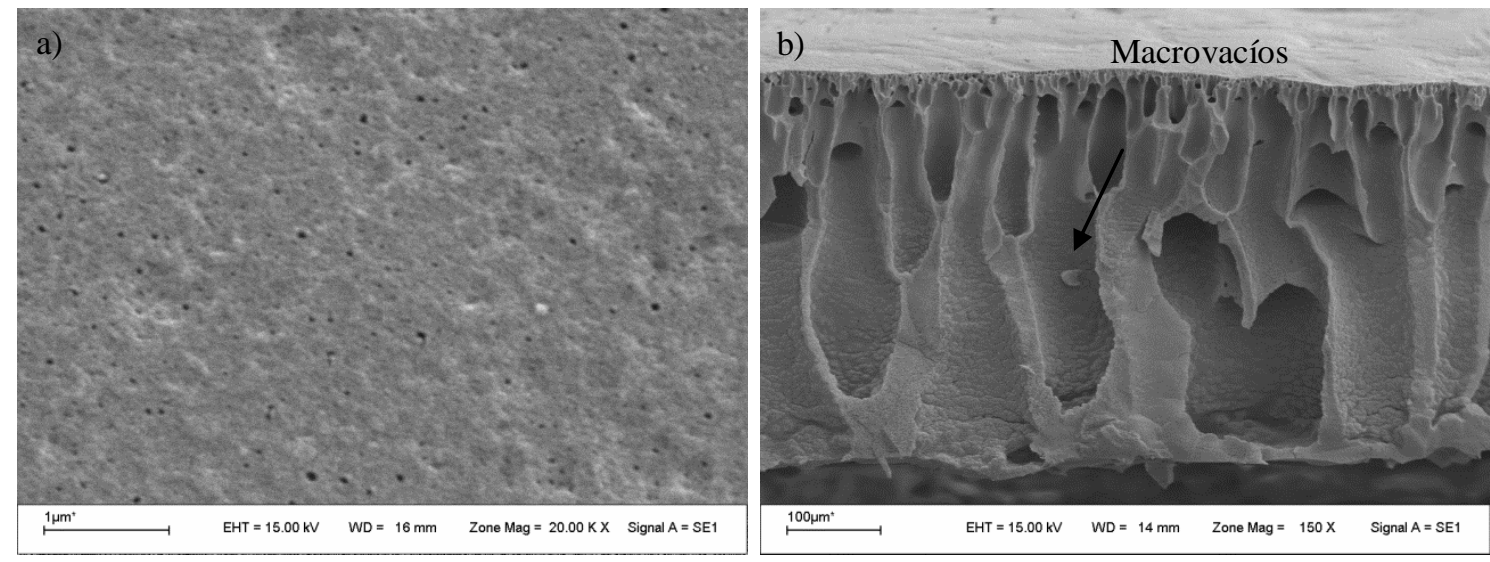

Figura 1: Micrografía SEM: a) corte superficial, b) corte transversal

\subsection{Permeación de agua pura}

A partir de la Ley de Darcy (ec. 2), se determinó la permeabilidad hidráulica de la membrana virgen. La misma fue de $1001,4 \pm 18 \mathrm{Lm}^{-2} \mathrm{~h}^{-1}$ bar. Dicho valor fue referente para la limpieza de la membrana luego de cada experiencia.

\subsection{Permeación del efluente acuoso del lavado de BD}

Todas las experiencias de permeación, tanto del efluente sintético como del efluente acuoso real, se llevaron a cabo durante 90 minutos con el fin de determinar el comportamiento del flujo permeado en función del tiempo. En la Figura 2 se puede ver el comportamiento del flujo permeado en el tiempo para el efluente real. Inicialmente se produce una caída de flujo hasta que el sistema alcanza el estado estacionario. Dicho comportamiento puede ser atribuido al fenómeno de concentración por polarización, a la adsorción y/o al desarrollo de una capa gel en la superficie de la membrana. En la Figura 2 también puede observarse que al aumentar la presión, el flujo permeado disminuye. En las Tablas 1 y 2 también se hace evidente el efecto de la presión sobre el flujo permeado. 
Tabla 1: Resultados de permeación obtenidos a partir del efluente acuoso de biodiesel preparado

\begin{tabular}{|c|c|c|c|c|c|c|}
\hline $\mathrm{V}(\mathrm{m} / \mathrm{s})$ & $\Delta p$ (bar) & $\mathrm{J}^{60}\left(\mathrm{~L} / \mathrm{hm}^{2}\right)$ & $\mathrm{DQO}_{\mathrm{P}}(\mathrm{ppm})$ & \%R DQO & $G \& A_{p}(m g / L)$ & \%R G\&A \\
\hline \multirow{3}{*}{0,5} & 0,4 & $60,7 \pm 1,2$ & 45682,7 & 39,87 & 0 & 100 \\
\hline & 0,6 & $53,3 \pm 1,2$ & 49092,5 & 35,38 & 0 & 100 \\
\hline & 1 & $51,7 \pm 0,9$ & 52315,4 & 31,14 & 0 & 100 \\
\hline \multirow{3}{*}{1} & 0,4 & $86,4 \pm 1,5$ & 48640,6 & 35,97 & 0 & 100 \\
\hline & 0,6 & $74,8 \pm 1,8$ & 50457.4 & 33,58 & 0 & 100 \\
\hline & 1 & $63,2 \pm 2,1$ & 54097,7 & 28,79 & 0 & 100 \\
\hline
\end{tabular}

$\mathrm{DQO}_{\mathrm{A}}=75971,2 \mathrm{ppm} ; \mathrm{G} \& \mathrm{~A}_{\mathrm{A}}=1850 \mathrm{mg} / \mathrm{L}$

Tabla 2: Resultados de permeación obtenidos a partir del efluente acuoso de biodiesel real

\begin{tabular}{lllllll}
\hline $\mathbf{V}(\mathbf{m} / \mathbf{s})$ & $\Delta \mathbf{p}(\mathbf{b a r})$ & $\mathbf{J}^{60}\left(\mathbf{L} / \mathbf{h m}^{2}\right)$ & $\mathbf{D Q O}_{\mathbf{P}}(\mathbf{p p m})$ & \%R DQO & G\&AP $_{(\mathbf{m g} / \mathbf{L})}$ & \%R G\&A \\
\hline \multirow{2}{*}{0,5} & 0,4 & $63,9 \pm 1,9$ & 49353,5 & 28,25 & 50 & 97,22 \\
& 0,6 & $44,4 \pm 1,3$ & 53454,2 & 22,28 & 100 & 94,44 \\
& 1 & $43,5 \pm 1,6$ & 55083,8 & 19,92 & 100 & 94,44 \\
\hline \multirow{2}{*}{1} & 0,4 & $83,5 \pm 1,3$ & 51757,6 & 24,75 & 200 & 88,89 \\
& 0,6 & $69,4 \pm 2,0$ & 54928,7 & 20,14 & 150 & 91,67 \\
& 1 & $51,0 \pm 1,1$ & 55230,3 & 19,70 & 150 & 91,67
\end{tabular}

$\mathrm{DQO}_{\mathrm{A}}=68783,6 \mathrm{ppm} ; \mathrm{G} \& \mathrm{~A}_{\mathrm{A}}=1800 \mathrm{mg} / \mathrm{L}$

Este fenómeno puede deberse a que a medida que aumenta la presión, el transporte del soluto hacia la superficie de la membrana aumenta, lo que produce un aumento o acumulación de soluto en la interface de la misma. A medida que la presión es mayor puede forzar la penetración de soluto a través de los poros de la membrana, y ocasionando la disminución del coeficiente de retención. Debido al efecto de polarización por concentración se produce una disminución en el flujo permeado. De todas maneras, es de hacer notar que las grasas y aceites son casi totalmente retenidas por la membrana en ambas alimentaciones, las cuales impactan de manera importante al valor de DQO.

Estos resultados son coincidentes con los obtenidos por GONCALVEZ et al. [19], quienes lograron eliminar en una primera etapa, mediante filtración con membranas, sólidos suspendidos, turbidez, aceites y grasas.

Puede evidenciarse que, para lograr mejores resultados en el tratamiento de estos efluentes, se debería implementar un proceso híbrido con al menos dos etapas. En una primera etapa, mediante filtración por membranas, se eliminarían grasas y aceites y sólidos suspendidos, y luego con una segunda etapa, implementando una tecnología tradicional tal como adsorción, se logre reducir el contenido de DQO. 


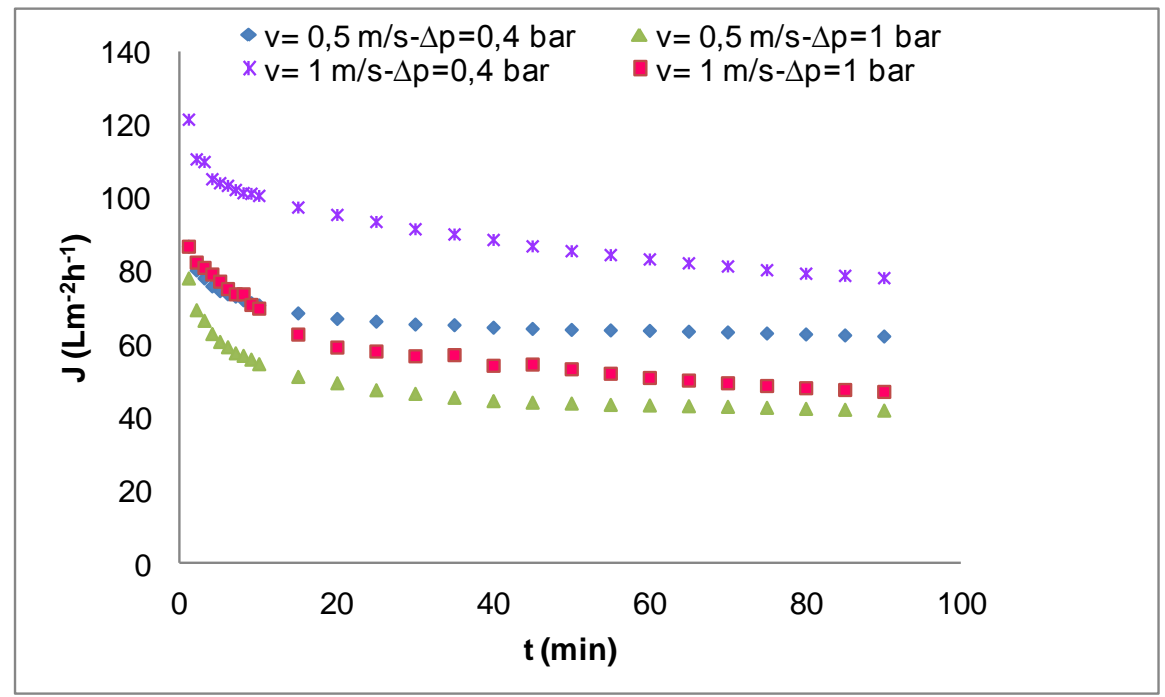

Figura 2: Flujo de efluente real provenientes de la industria de biodiesel permeado.

Diferentes autores han informado este tipo de comportamiento para emulsiones aceite-agua. HUA et al. [20] estudiaron el proceso de MF de flujo cruzado en aguas residuales oleosas utilizando membranas cerámicas de alúmina. Encontraron que un aumento en la presión de transmembrana produjo efectos positivos y negativos sobre el flujo de permeado. Entre 0,05-0,2 MPa el flujo de permeado presento una leve disminución, manteniéndose prácticamente constante. Mientras que entre 0,2-0,3 MPa, el flujo de permeado descendió de manera brusca. En este rango, una mayor presión permitió que las gotitas (del disolvente y del soluto) pasaran más rápidamente a través de los poros de la membrana. Sin embargo, las gotitas de aceite se acumularon en la superficie y en los poros de la membrana, produciendo incrustaciones. Los autores sostienen que a una excesiva presión, la polarización del gel causa una disminución del flujo; y en base a estos resultados sugieren que 0,2 $\mathrm{MPa}$ (2 bares) es la presión adecuada para trabajar con este tipo de sistema.

Los TSS de la muestra real fueron de $\sim 6 \%{ }^{\circ}$ Brix, mientras que los de la muestra sintética fueron $\sim 4{ }^{\circ}$ Brix TSS se observó que los mismos no fueron retenidos por la membrana.

El pH de la muestra real fue de $\sim 5,5$ mientras que el $\mathrm{pH}$ de la muestra sintética fue de $\sim 6$.

En la Figura 3 se muestra una imagen de la alimentación y permeado obtenidos en las experiencias de UF utilizando efluente sintético y real.

Se puede observar claramente una diferencia entre el permeado y la alimentación en ambos casos, debido a la retención de grasa y aceites. Sin embargo, entre ambas soluciones, se observa que la alimentación sintética es una mezcla más homogénea que la real. A pesar de que estas muestras poseen similares composiciones, existe una diferencia del $10 \%$ en el contenido de DQO. Esto puede deberse a que la muestra sintética posee $50 \mathrm{mg} / \mathrm{L}$ más de G\&A. Evidentemente, pequeñas variaciones en el contenido de G\&A afectan de manera sensible a la retención de DQO.
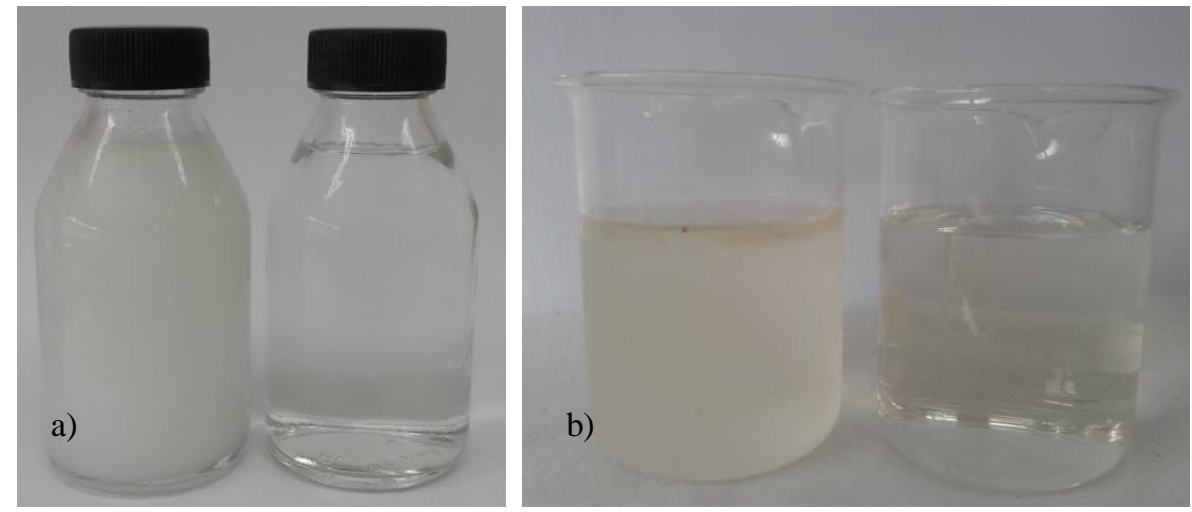

Figura 3: a) Alimentación y permeado de efluente sintético, b) alimentación y permeado de efluente real. 


\section{CONCLUSIONES}

En función de los resultados experimentales obtenidos, se arribó a las siguientes conclusiones:

- Todas las experiencias de permeación del efluente acuoso (tanto el preparado como el real) mostraron en los primeros minutos, un decaimiento del flujo permeado en función del tiempo hasta hacerse estable. Este comportamiento es atribuido al fenómeno de polarización por concentración y formación de capa de gel.

- Al aumentar la velocidad de permeación a presión constante, el flujo permeado aumenta gradualmente, ya que al aumentar la velocidad se logra limpiar la superficie de la membrana obteniendo un menor ensuciamiento

- La membrana estudiada retiene entre el $89-100 \%$ de G\&A, lo que disminuyó notablemente la turbidez del efluente

- El descenso en la corriente de permeado de la Demanda Química de Oxígeno fue del 40\% respecto a la alimentación.

- Tanto los valores de $\mathrm{pH}$ como de TSS no se afectaron por el tratamiento.

\section{AGRADECIMIENTOS}

Los autores agradecen al Consejo Nacional de Investigación de Argentina (CONICET) y a la Universidad Nacional de Río Cuarto (UNRC).

\section{BIBLIOGRAFÍA}

[1] HILBERT, J. H., SBARRA, R., LÓPEZ AMORÓS, M. Producción de Biodiesel a partir de aceite de soja. Contexto y evaluación reciente, INTA. Ministerio de Agricultura, Ganadería y Pesca. Presidencia de la Nación., Buenos Aires, 2012.

[2] ALVES, M.J., NASCIMENTO, S.M., GOMES PEREIRA, I., et al., "Biodiesel purification using micro and ultrafiltration membranes". Renewable Energy, v. 58, pp. 15-20, Oct. 2013.

[3] ATADASHI, I.M., AROUA, M.K., ABDUL AZIZ, A.R., et al., "The effects of water on biodiesel production and refining technologies: A review", Renewable and Sustainable Energy Reviews, v. 16, pp. 34563470, Apr. 2012.

[4] SUEHARA, K., KAWAMOTO, Y., FUJII, E., et al., "Biological treatment of wastewater discharged frombiodiesel fuel production plant with alkali-catalyzed transesterification". Journal of Bioscience and Bioengineering, v. 100 , n.4, pp. 437-442, Nov. 2005.

[5] LOW, S.C., GAN, G.K., CHEONG, K.T. "Separation of Methyl Ester from Water in a Wet Neutralization Process", Journal of Sustainable Energy \& Environment, v. 2, n. 1, pp. 15-19, Jan. 2011.

[6] MEHRABADI, A., CRAGGS, R., FARID, M.M. "Biodiesel production potential of wastewater treatment high rate algal pond biomass", Bioresource Technology, v. 221, pp. 222-233, Sep.2016.

[7] DEMIRBAS, E., KOBYA, M., "Operating cost and treatment of metalworking fluid wastewater by chemical coagulation and electrocoagulation processes.Process Safety and Environment Protection". Process Safety and Enviromental Protection, v. 105, pp. 79-90, Jan. 2017.

[8] DAUD, N.M., ABDULLAH, S.R.S., HASAN, H.A., et al., "Production of biodiesel and its wastewater treatment technologies: A review". Process Safety and Environmental Protection, v. 94, pp. 487-508, Mar. 2015.

[9] DAUD, Z., AWANG, H., AB AZIZ ABDUL LATIF, A.A.A., et al.,"Suspended Solid, Color, COD and Oil and Grease Removal from Biodiesel Wastewater by Coagulation and Flocculation Processes", Procedia - Social and Behavioral Sciences, v. 195, pp. 2407-2411, Jul. 2015.

[10] NGAMLERDPOKIN, K., KUMJADPAI, S., CHATANON, P., et al., "Remediation of biodiesel wastewater by chemical- and electro-coagulation: A comparative study", Journal of Environmental Management, v. 92, pp. 2454-2460, Jun. 2011.

[11] PITAKPOOLSIL, W., HUNSOM, M. "Adsorption of pollutants from biodiesel wastewater using chitosan flakes", Journal of the Taiwan Institute of Chemical Engineers, v. 44, pp. 963-971, Apr. 2013. 
[12] PITAKPOOLSIL, W., HUNSOM, M. "Treatment of biodiesel wastewater by adsorption with comercial chitosan flakes: Parameter optimization and process kinetics”. Journal of Environmental Management, v. 133, pp. 284-292, Ene. 2014.

[13] SHARMA, Y.C., SINGH, B., UPADHYAY, S.N. "Advancements in development and characterization of biodiesel: A review”, Fuel, v. 87, pp. 2355-2373, Sep. 2008.

[14] SHIRAZI, M.M.A., KARGARI, A., BAZGIR, S., et al., "Characterization of electrospun polystyrene membrane for treatment of biodiesel's water-washing effluent using atomic force microscopy", Desalination, v. 329, pp. 1-8, Sep. 2013.

[15] MARCHESE, J., OCHOA, N.A., PAGLIERO, C., et al., "Pilot-Scale Ultrafiltration of an Emulsified Oil Wastewater”. Environmental Science \& Technology, v. 34, n. 14, pp. 2990-2996, Jun. 2000.

[16] MASUELLI, M., MARCHESE, J., OCHOA, N.A. "SPC/PVDF membranes for emulsified oily wastewater treatment”, Journal of Membrane Science, v. 326, pp. 688-693, Ene. 2009.

[17] KESTING, R. "Synthetic Polymeric Membranes”, 2 ed., New York, USA, 1985.

[18] FIRMAN, L., PAGLIERO, C., OCHOA, N.A., et al., "PVDF/PMMA membranes for lemon juice clarification: fouling analysis", Desalination and Water Treatment, v. 55, pp. 1167-1176, Jun. 2014.

[19] GONÇALVEZ, B. R., MACHADO, A. E. H., TROVÓ, A. "Treatment of a biodiesel effluent by coupling coagulation-flocculation, membrane filtration and Fenton reactions", Journal of Cleaner Production, $v$. 142, pp. 1918-1921, Jan. 2017.

[20] HUA, F.L., TSANG, Y.F., WANG, Y.J., et al., "Performance study of ceramic microfiltration membrane for oily wastewater treatment", Chemical Engineering Journal, v. 128, pp. 169-175, Apr. 2007. 